\title{
When you dislike patients, pain is taken less seriously
}

Lies De Ruddere ${ }^{1}$, Liesbet Goubert ${ }^{1}$, Ken Martin Prkachin ${ }^{2}$, Michael André Louis Stevens ${ }^{3}$, Dimitri Marcel Leon Van Ryckeghem ${ }^{1}$, Geert Crombez ${ }^{1}$

${ }^{1}$ Department of Experimental-Clinical and Health Psychology, Ghent University, Ghent, Belgium

${ }^{2}$ University of Northern British Columbia, Prince George, British Columbia, Canada

${ }^{3}$ Department of Data Analysis, Ghent University, Ghent, Belgium

Corresponding author: Lies De Ruddere, Department of Experimental-Clinical and Health Psychology, Ghent University, Henri Dunantlaan 2, B-9000 Gent, Belgium. Tel: +32 (0)9 264

86 11, Fax: +32 (0)9 26464 89. Electronic mail may be sent to Lies.DeRuddere@ Ugent.be.

Text pages: 20

Figures: 2

Tables: 0

Keywords: pain estimation; patients' likability; pain expression; observers 


\section{Introduction}

Pain is a prevalent health problem [7], entailing severe personal and social impacts [3] as well as financial costs [8]. However, pain management often remains inadequate [34]. One important aspect of pain management is the estimation of pain by observers, as potential caregivers [15]. Others observing a person in pain can vary in the amount of pain they impute to a sufferer. It is reasonable to assume that such differences influence the responses to the sufferer, such as treatment choices or helping behavior in the everyday social environment. Hence, insight into how pain estimations originate is essential.

Some variables have been found to have a major impact upon pain estimation. Factors related to the sufferer are the expression of pain $[14,41]$ or the physical attractiveness of the pain sufferer $[16,17,18]$. Factors related to the observer, are observers' catastrophizing about (the sufferer's) pain [14,35], or observers' past experience with pain of others [28]. Also contextual factors play a role, such as the presence of a medical cause for pain $[4,5,36,37]$. The present study focuses on one factor that is important in clinical practice, i.e. patient's likability. Next to perceived treatability and manageability, perceived likability (i.e., the degree to which the patient is liked by an individual) contributes to the perception of patients' characteristics $[32,42]$. Previous studies, using vignettes, have demonstrated that observers attribute more severe symptoms (i.e., more pain, distress and disability) to liked than to disliked patients $[4,36,37]$. However, pain estimations are likely the result of a combination of factors related to the observer and factors related to the patient $[12,13]$. The study aims to extend existing research by using videos of real patients, in order to investigate whether the effects of likability of patients are dependent upon the level of pain expressed by patients.

Insight into the processes underlying pain estimations is important [28,30]. Pain estimation might reflect two processes: observers may be sensitive to a patient's pain (i.e., 
being able to discriminate between levels of pain), and/or have a general tendency to ascribe pain to a patient (i.e., response bias) without taking into account a patient's pain cues. Insight into these two processes is important as they might have implications for clinical practice.

In the present study, an evaluative conditioning procedure (EC) [19] was used to manipulate the likability of patients, by associating pictures of patients with either positive, neutral or negative personal traits. Videos of the patients were then presented to healthy volunteers (observers) who rated patients' pain. We expect observers (1) to rate the pain of liked patients as more intense than the pain of disliked patients (primary outcome; pain ratings on VAS), (2) to be more sensitive to pain expressed by liked patients than to pain expressed by disliked patients (sensitivity; possible mechanism), and (3) to have a higher tendency to ascribe pain to liked than to disliked patients (response bias; possible mechanism). Furthermore, we explored whether the level of pain expressed by patients moderated the effect of patients' likability upon observers' pain estimations.

\section{Methods}

\subsection{Participants}

Participants were recruited by means of an advertisement in local newspapers. To be eligible, participants had to be 18 years or older, and had to speak the Dutch language fluently. Potential participants who reported a current psychiatric disorder were excluded. Forty healthy volunteers (17 men and 23 women) participated. Mean age was 35.20 years (SD $=14.55$; range $=19-65$ years). Participants were rewarded $€ 15$. All participants were Caucasian. The study was approved by the ethical committee of the Faculty of Psychology and Educational Sciences of Ghent University. 


\subsection{Design}

The experiment consisted of two phases. In the first phase, adjectives denoting positive, neutral or negative personal traits (unconditioned stimuli; UCs) were paired with pictures of six different patients (conditioned stimuli; CSs), by means of an evaluative conditioning (EC) procedure. EC is a procedure in which a change in the valence of a stimulus (CS) is realized due to the pairing of that stimulus with another positive or negative stimulus (UCS) $[9,10]$. In the second phase, video fragments of the same patients performing pain-inducing activities were shown to the participants, who were asked to rate the pain of the patients.

\subsection{Apparatus and stimuli}

The experiment was programmed and presented by the Inquisit Millisecond software package [20] on a 745 Dell Optiplex computer with a $75 \mathrm{~Hz}, 19$ inch color CRT monitor. Pictures of faces of six patients (three men) with shoulder pain were used as conditioned stimuli (CSs). These pictures were obtained by means of a screenshot from the UNBCMcMaster Shoulder Pain Archive [29]. Unconditioned stimuli consisted of nine Dutch adjectives (personal traits). These adjectives describe personal traits that are rated on the degree to which they have good or bad consequences for others dealing with the possessors of the traits. The ratings range from 100 (extremely negative) through 500 (neutral) to 900 (extremely positive) [see 25 for the scaling method]. Three words with a positive valence [faithful $(M=820, S D=83)$, honest $(M=815, S D=99)$, friendly $(M=760, S D=94)]$, three with a negative valence [egoistic $(M=180, S D=62)$, hypocritical $(M=185, S D=81)$, arrogant $(M=240$, 
$S D=99)$ ] and three with a neutral valence [true to tradition $(M=510, S D=102)$, reserved $(M=495, S D=128)$, conventional $(M=480, S D=128)]$ were selected.

During the rating phase, a set of video fragments of these patients was shown to participants. These video fragments were selected from a set of videos displaying facial pain expressions of shoulder pain patients undergoing a physiotherapy assessment protocol [29]. For the present study, 8 videos of 6 different patients were selected, resulting in 48 video fragments. As it was not possible to obtain video fragments ranging from no to high intensity pain expression for each of the 6 patients, two different patients (one male and one female) were selected for each category (no, mild and high intensity pain expression). Pain scores were on a composite index based on the intensity of four facial actions that have been associated with pain $[27,31]$. The scores can range from $0-16$. Scores of 0 were taken to define no pain. Scores of 3-6 defined mild pain and scores of 7 or higher defined high pain Each fragment had a length of 2000 milliseconds (ms).

\subsection{Questionnaires}

\subsubsection{Pain estimation}

A $100 \mathrm{~mm}$ visual analogue scale was used to rate pain of the patients. The endpoints of the scale were marked by 'no pain' on the left and by 'pain as bad as could be' on the right.

\subsubsection{Manipulation check questionnaire.}

The effectiveness of the evaluative conditioning manipulation was checked by means of three 21-point scales. The scales measured the extent to which the participant judged the patient to be negative or positive $(-10=$ very negative, $0=$ neutral, $10=$ very positive $)$, disagreeable or agreeable $(-10=$ very disagreeable, $0=$ neutral, $10=$ very agreeable $)$, and 
unsympathetic or sympathetic $(-10=$ very unsympathetic, $0=$ neutral, $10=$ very sympathetic $)$. A mean score for likability of the patients was calculated by averaging the scores on the three questions.

\subsection{Procedure}

\subsubsection{Preparation.}

In the experiment room, the participant was seated at about $60 \mathrm{~cm}$ from the computer screen. To minimize demand effects, the following cover story was used: participants were informed that this study examined spontaneous psychophysiological responses (e.g. heartbeat, respiration, skin conductance) of people observing other persons in pain. Furthermore, each participant was told that numerous variables influence these psychophysiological reactions and that the present study focused on one specific factor: the verbal information about the observed person. Next, the course of the experiment was shortly explained; i.e. participants were told that (1) verbal information about six different persons would be given and (2) they would be asked to observe several video fragments of these persons and rate their pain. The first written informed consent was obtained. Electrodes and a respiration strain gauge for the psychophysiology measurement (there was no real record of the psychophysiology) were applied.

\subsubsection{Acquisition phase.}

By means of instructions presented on the computer screen, participants were informed that pictures of six different patients would be presented together with some information about those patients. Participants were asked to pay close attention to the information. When the participant pressed the ENTER button, stimuli (CS-US pairs) were 
randomly presented. For each participant, one male and one female patient were always associated with positive words, one male and one female patient with neutral words, and one male and one female patient with negative words. This pattern was counterbalanced between participants. The CS was presented for $2000 \mathrm{~ms}$ followed by the presentation of the US together with the CS, for $3000 \mathrm{~ms}$. After each CS-US pair, there was an inter trial interval of 1000 ms (see Fig. 1). Each CS-US pair appeared 9 times. In sum, 54 trials were presented to each participant.

\section{INSERT FIGURE 1 ABOUT HERE}

\subsubsection{Rating phase.}

The 48 video fragments were each three times randomly presented on the computer screen. Each participant received a different random sequence of video fragments. After a video fragment $(2000 \mathrm{~ms})$ was shown, a black screen appeared. At this moment, the participant indicated by means of a vertical line on the VAS how much pain he/she thought the person was experiencing. The black screen disappeared when the participant pressed the ENTER button, initiating the next video fragment.

\subsubsection{Manipulation check.}

The pictures of the six patients were presented again. For each picture, participants indicated their current evaluation of the person. Presentation of pictures was randomized across participants. At the end of the study, the participant filled in a second informed consent, which revealed the true purpose of the study. 


\subsection{Data reduction and statistical analysis}

\subsubsection{Sensitivity and response bias.}

By means of signal detection analyses, two measures were calculated. First, observers' sensitivity, i.e. the ability to discriminate between levels of patients' pain expression, was measured by calculating the nonparametric index $P(A)$ [24]. In particular, observers' ability to differentiate between no and mild intensity pain, between no and high intensity pain and between mild and high intensity pain was measured. $P(A)$ is the average of all the maximum and minimum areas under the Receiver Operating Characteristic defined by observers' performance [see 24 for the method to calculate $P(A)$ ]. Values of $P(A)$ can range from 0 to 1.0 , with .5 indicating chance performance or a lack of ability to discriminate between levels of patients' pain expression. Second, observers' response bias, i.e. the overall likelihood to attribute pain to the patient, was evaluated by calculating the nonparametric index $B$ [24]. $B$ is the point on the visual analogue scale at which the observer is equally likely to refer to a higher or a lower pain expression. The higher the $B$ value, the higher the overall likelihood of attributing pain to a patient, irrespective of the level of patients' pain expression.

\subsubsection{Statistical analyses.}

Analyses were conducted for the following dependent variables: the scores on the manipulation check questionnaire, the pain ratings, the sensitivity scores $(P(A))$, and the response bias scores $(B)$. In the manipulation check analysis, valence of the traits (positive, neutral or negative) that was combined with the patients in the acquisition phase was the only factor (we will refer to this factor as 'valence of traits'). In the pain rating analysis, there were two factors: 'valence of traits' and patient's 'pain expression' (no pain, mild intensity pain or high intensity pain). In the sensitivity score analysis, there were also two factors: 'valence of 
traits' and the two levels of pain expression between which the observer had to discriminate. This factor had three levels: discrimination between no and mild intensity pain, between no and high intensity pain or between mild and high intensity pain (we will refer to this factor as 'discrimination between levels of pain'). 'Valence of traits' was the only factor in the response bias analysis.

The factors in the pain rating study were manipulated partially within and partially between subjects. Within subjects, each level of 'valence of traits' was combined with only two of the three levels of 'pain expression'. Between subjects, every level of 'valence of traits' was combined with every level of 'pain expression'. Therefore, we analyzed the results using linear mixed effects models as implemented in the $\mathrm{R}$ package nlme [26]. This is a commonly used alternative to repeated measures analysis that can handle a wider variety of designs [26]. In short, linear mixed effects models account for the correlations in withinsubjects data by estimating subject-specific deviations (or random effects) from each population-level factor (or fixed factor) of interest [see 40 for an elaboration].

Each analysis consisted of three steps. First, all relevant factors and interactions were entered in the model as fixed factors. In the second step, we assessed whether it was necessary to add a random effect for each of the fixed factors in the analysis. If a random effect increased the fit of the model it was included in the final model. In the third step, we inspected the ANOVA table of the final model and tested specific hypotheses about possible main effects or interactions [see 39 for a similar approach]. When testing specific hypotheses, standardized regression weights were reported as a measure of effect size.

\section{Results}

\subsection{Manipulation check}


In this analysis, the random effect of 'valence of traits' was necessary. A significant main effect of 'valence of traits' was found $(F(2,78)=15.98, p<.001)$ : patients associated with negative traits were rated as less likable than patients associated with neutral traits $(F(1,78)=20.44, p<0.001 ; \beta=-.76)$. Patients associated with neutral traits were rated as less likable than patients associated with positive traits $(F(1,78)=10.22, p<.01 ; \beta=-.49)$.

\subsection{Pain ratings}

In this analysis, both the random effects of 'pain expression' and 'valence of traits' were necessary. A significant main effect of 'pain expression' was found $(F(2,5709)=83.49$, $p<.001)$. Observers attributed more intense pain to patients expressing more pain: pain ratings were higher in the mild pain expression condition than in the no pain expression condition $(\mathrm{F}(1,5709)=71.87, p<.001, \beta=0.68)$ and higher in the high pain expression condition than in the mild pain expression condition $(\mathrm{F}(1,5709)=94.71, p<.001, \beta=0.58)$. The main effect of 'valence of traits' was not significant $(F(2,5709)=1.38, p=.25)$. However, the interaction between 'valence of traits' and 'pain expression' was significant $(F(2,5709)=13.00, p<.001)$. In the high pain expression condition, the effect of 'valence of traits' was significant $(F(2,5709)=10.63, p<.001)$. Observers' pain ratings of patients who were presented with negative traits were significantly lower than pain ratings of patients who were presented with neutral $(F(1,5709)=10.23, p<.01, \beta=-.26)$ or positive traits $(F(1,5709)$ $=19.39, p<.001 . \beta=-.32$ ). Observers' pain ratings of patients that were presented with positive and neutral traits did not differ significantly $(F<1)$ (see Fig. 2). Both in the no pain $(F(2,5709)=1.52, p=.22)$ and the mild pain condition $(F(2,5709)=1.43, p=.23)$ there was no effect of 'valence of traits'. 


\section{INSERT FIGURE 2 ABOUT HERE}

\subsection{Sensitivity and response bias}

In the sensitivity analysis, only the random effect of 'discrimination between levels of pain' was necessary. The variable 'valence of traits' $(F(2,72)=9.27, p<.001)$ had a significant effect upon perceptual sensitivity, indicating lower sensitivity to pain of patients who were presented with negative traits than to pain of patients who were presented with neutral $(F(1,72)=10.81, p<.01, \beta=-.55)$ or positive traits $(F(1,72)=16.09, p<.001, \beta=-$ .63). Sensitivity to pain of patients who were presented with neutral traits did not differ from sensitivity to pain of patients who were presented with positive traits $(F<1)$. Furthermore, a main effect of 'discrimination between levels of pain' was found $(F(2,72)=8.50, p<.001)$, demonstrating better ability to discriminate no pain from high intensity pain than no pain from mild intensity pain $(F(1,72)=9.99, p<.01, \beta=.49)$ or mild intensity from high intensity pain $(F(1,72)=15.88, p<.001, \beta=.80)$. The ability to discriminate mild intensity from high intensity pain did not differ from the ability to discriminate no from mild intensity pain $(F(1,72)=3.63, p<.06)$. The interaction effect between 'valence of traits' and 'discrimination between levels of pain' was not significant $(F<1)$. In the response bias analysis no effect of 'valence of the traits' upon response bias scores was found $(F<1)$.

\section{Discussion}

The present study investigated the influence of patients' likability upon observers' pain estimations, sensitivity towards pain and response bias. Patients' likability was 
manipulated by means of an evaluative conditioning procedure in which a change in the valence of a stimulus (the patient) was realized due to the pairing of that stimulus with another positive or negative stimulus (i.e., adjectives describing personal traits) $[9,10]$. In the present study, patients associated with negative traits were rated as less likable than patients associated with neutral traits and patients associated with neutral traits were rated as less likable than patients associated with positive traits. It was found that pain of disliked patients expressing high intensity pain was estimated as less intense than pain of liked patients expressing high intensity pain. Furthermore, observers were less sensitive towards pain of negatively evaluated patients than to pain of positively evaluated patients. No influence of patients' likability upon response bias was found.

The findings are in line with the findings of Chibnall and Tait (1995) [4] and Tait and Chibnall (1994; 1997) [36,37], indicating higher pain, disability and distress scores attributed to liked than to disliked patients. Moreover, the present study extends this research by using real instead of fictitious patients, enabling to examine the interaction between top-down (i.e., patients' likability) and bottom-up factors (i.e., patients' facial expressions). Noteworthy, the effect of patients' likability was only found for patients expressing high intensity pain. This seems to be paradoxical as we would expect contextual information to be the most influential with regard to ambiguous stimuli (mild intensity pain). However, the high levels of pain expression could have induced feelings of suspiciousness in the observers as extreme levels of pain expression are characteristic of faked or exaggerated pain [6]. According to Kahneman (2003) [21], we could expect that the observers - when feeling suspiciousness about the realness of the pain symptoms - made use of the contextual information (i.e. patients' likability) to facilitate the pain judgment. The expression of mild and no pain, to the contrary, might not have contributed to suspiciousness and might have served as reliable and sufficient cues to make the pain estimation. 
Although we did not find a main effect of patients' likability upon pain estimations, we did find a main effect upon observers' sensitivity for the pain. These results extend - on a behavioral level - neurological findings in the context of sensitivity for pain. It has been shown, for example, that the neural network activated in an observer of someone in pain, is highly similar to the neural network activated in the person in pain himself [2]. This perception-action coupling - the activation of a representation of a behavior that is observed in someone else - is less pronounced when observers dislike the observed person in pain [33]. Further, we did not find an effect of patients' likability upon observers' response bias, indicating that observers are not more inclined to attribute pain to positive than to negative patients - irrespective of patients' pain expression. This parallels the finding that there was no main effect of patients' likability upon pain estimations.

Identifying variables that influence pain estimation by others is relevant as pain estimation might influence crucial actions concerning pain management both in the professional context as well as in the everyday environment. Our results suggest that pain of disliked patients who express high pain is taken less seriously by others. This could imply less helping behavior by others as well as poorer health outcomes. However, research into consequences of reduced pain estimation is lacking. Further, research into factors which might be responsible for the likability of a pain patient is worthwhile as well. For example, there is evidence that observers have more negative attitudes towards patients when medical evidence for the pain is lacking [4] or when the duration of the pain is chronic $[11,38]$.

In addition, the question remains why observers estimate pain to a lower degree and are less sensitive to pain when they do not like the patient. Hadjistavropoulos and colleagues $[16,17,18]$, for example, found that observers perceived unattractive patients as experiencing more pain than attractive patients. At first sight this seems at odds with our findings. However, it is important to distinguish between physical unattractiveness and unattractiveness 
due to personal traits. Physically unattractive patients may be perceived by others as unhealthy due to their physical appearance. This reasoning may not apply to attractiveness based upon personal traits. From an evolutionary perspective, attractiveness based upon personal traits (or patients' likability) may have different effects [41]. Taking an evolutionary perspective on helping behavior [41], observers of others in pain may optimize their provision of care by being alert to social cheating, and only deliver help to those who are in real pain. Observing disliked persons in pain could activate the cheating detection mechanism. Being sensitive towards the pain of 'negative' persons, would not be expected to have any benefits to the observer and the social exchange would not be warranted [22,23]. In our study, only the attractiveness with regard to personal traits (patients' likability) was manipulated. As facial expressions were counterbalanced across the valences of the personal traits, physical attractiveness could not have influenced our results.

There are some limitations to this study. First, as in most vignette studies on this topic [see 4,36], observers were lay people. One should, therefore, be cautious in generalizing results towards professional caregivers. Tait and Chibnall (1997) [37] suggest for example that professional caregivers might interpret negative traits of patients more diagnostically, i.e., for example, as a consequence of the pain they feel. Future studies should investigate the influence of patients' likability upon the estimation of patients' pain by professional caregivers. Second, observers only saw the facial expressions of patients. Future research would benefit from including information on full body movements instead of only facial expressions, as this can be considered more ecologically valid. Finally, we opted for a procedure that resulted in awareness of the contingencies between CS and UCS. This awareness could have induced demand effects. However, to reduce demand effects, we used a cover story that has previously been used in studies on evaluative conditioning [e.g., 1]. At the 
end of the experiment, none of the participants was able to identify the true purpose of the study.

To conclude, this study provides evidence for the impact of patients' likability upon estimation of pain by others. Pain of disliked patients was found to be taken less seriously than pain of liked patients. Further research into the underlying mechanisms, as well as into the consequences of reduced pain estimation of disliked patients is needed. Also, replication of the data with professional caregivers and with other pain behavior is recommended.

\section{Acknowledgments}

This study was supported by a grant from the Fund for Scientific Research (FWO) - Flanders (G.0299.09; G.0178.07N). The authors would like to thank Catherine De Koker for her help with data collection and input of the data. There are no conflicts of interest that may arise as a result of the research presented in this manuscript. 


\section{References}

[1] Baeyens F, Hermans D, Eelen P. The role of CS-US contingency in human evaluative conditioning. Behav Res Ther 1993;31:731-737.

[2] Botvinick M, Jha AP, Bylsma LM, Fabian SA, Solomon PE, Prkachin KM. Viewing facial expressions of pain engages cortical areas involved in the direct experience of pain. Neuroimage 2005;25:312-319.

[3] Breivik H, Collet B, Ventafridda V, Cohen R, Gallacher D. Survey of chronic pain in Europe: prevalence, impact on daily life, and treatment. Eur J Pain 2006;10:287-333.

[4] Chibnall JT, Tait RC. Observer perception of low back pain: effects of pain report and other contextual factors. J Appl Soc Psychol 1995;25:418-439.

[5] Chibnall JT, Tait RC. Social and medical influences on attributions and evaluations of chronic pain. Psych Health 1999;14:719-729.

[6] Craig KD, Hyde SA, Patrick CJ. Genuine, suppressed and faked facial behavior during exacerbation of chronic low-back-pain. Pain 1991;46:161-171.

[7] Crombie IK, Croft PR, Linton SJ, LeResche L, Von Korff M. Epidemiology of pain. Seattle: IASP Press, 1991.

[8] Dagenais S, Caro J, Haldeman S. A systematic review of low back pain cost of illness studies in the United States and internationally. Spine J 2008;8:8-20.

[9] De Houwer J. A conceptual and theoretical analysis of evaluative conditioning. Span J Psychol 2007;10:230-241.

[10] De Houwer J, Thomas S, Baeyens F. Associative learning of likes and dislikes: a review of 25 years of research on human evaluative conditioning. Psychol Bull 2001;127:853869. 
[11] Eccleston C, Crombez G. Worry and chronic pain: a misdirected problem solving model. Pain 1997;132:233-236.

[12] Goubert L, Craig KD, Buysse A. Perceiving others in pain: experimental and clinical evidence on the role of empathy. In Ickes W, Decety J, editors. The Social Neuroscience of Empathy. Massachusetts: The MIT Press, 2009. pp.153-165.

[13] Goubert L, Craig KD, Vervoort T, Morley S, Sullivan MJL, Williams ACdeC, Cano A, Crombez G. Facing others in pain: the effects of empathy. Pain 2005;118:285-288.

[14] Goubert L, Vervoort T, Cano, AM, Crombez G. Catastrophizing about their children's pain is related to higher parent-child congruency in pain ratings: an experimental investigation. Eur J Pain 2009;13:196-201.

[15] Hadjistavropoulos HD, Craig KD. A theoretical framework for understanding self-report and observational measures of pain: a communications model. Behav Res Ther 2002;40:551-570.

[16] Hadjistavropoulos T, LaChapelle D, Hale C, MacLeod FK. Age- and appearance-related stereotypes about patients undergoing a painful medical procedure. The Pain Clinic $2000 ; 12: 25-33$

[17] Hadjistavropoulos T, McMurtry B, Craig KD. Beautiful faces in pain: biases and accuracy in the perception of pain. Pscychol Health 1996;11:411-420.

[18] Hadjistavropoulos HD, Ross MA, von Baeyer Cl. Are pysicicians' ratings of pain affected by patients’ physical attractiveness? Soc Sci Med 1990;31:69-72.

[19] Hofmann W, De Houwer J, Perugini M, Baeyens F, Crombez G. Evaluative conditioning in humans: a meta-analysis. Psychol Bull 2010;136:390-421.

[20] Inquisit 1.33. Computer software. Seattle, WA: Millisecond Software, 2002.

[21] Kahneman D. A Perspective on judgment and choice. Am Psychol 2003;58:697-720.

[22] Kappesser J, Williams ACdeC. Pain judgments of patients' relatives: examining the use 
of social contract theory as theoretical framework. J Behav Med 2008;31:309-317.

[23] Kappesser J, Williams ACdeC. Pain estimation: asking the right questions. Pain 2010;148:184-187.

[24] McNicol D. A primer of signal detection theory. London: George Allen and Unwin, 1972.

[25] Peeters G. Evaluative meanings of adjectives invitro and in context - some theoretical implications and practical consequences of positive-negative asymmetry and behavioral-adaptive concepts of evaluation. Psychol Belg 1992;32:211-231.

[26] Pinheiro JC, Bates DM. Mixed-effects models in S and S-PLUS. New York: SpringerVerlag, 2000.

[27] Prkachin KM. The consistency of facial expressions of pain: a comparison across modalities. Pain 1992;51:297-306.

[28] Prkachin KM, Rocha EM. High levels of vicarious exposure bias pain judgments. J Pain 2010;11:904-909.

[29] Prkachin KM, Solomon PE. The structure, reliability and validity of pain expression: evidence from patients with shoulder pain. Pain 2008;139:267-274.

[30] Prkachin KM, Solomon PE, Ross J. Underestimation of pain by health-care providers: towards a model of the process of inferring pain in others. Can J Nurs Res 2007;39:88106.

[31] Rocha E, Prkachin KM, Beaumont SL, Hardy C, Zumbo BD. Pain reactivity and illness behavior in kindergarten-aged children. J Ped Psychol $2003 ; 28: 47-57$.

[32] Rouse RA, Hamilton MA. Dentists evaluate their patients: an empirical investigation of preferences. J Behav Med 1991;14:637-648. 
[33] Singer T, Seymour B, O’Doherty JP, Stephan KE, Dolan RJ, Frith CD. Empathic neural responses are modulated by the perceived fairness of others. Nature 2006;439:466469.

[34] Spacek A. Modern concepts of acute and chronic pain management. Biomed Pharmacother 2006;60:329-335.

[35] Sullivan MJL, Martel MO, Tripp DA, Savard A, Crombez G. Catastrophic thinking and heightened perception of pain in others. Pain 2006;123:37-44.

[36] Tait RC, Chibnall JT. Observer perceptions of chronic low back pain. J Appl Soc Psychol 1994;24:415-431.

[37] Tait RC, Chibnall JT. Physician judgments of chronic pain patients. Soc Sci Med 1997;45:1199-1205.

[38] Taylor AG, Skelton JA, Butcher J. Duration of pain condition and physical pathology as determinants of nurses' assessments of patients in pain. Nurs Res 1984;33:48.

[39] Verbruggen F, Aron AR, Stevens MA, Chambers CD. Theta burst stimulation dissociates attention and action updating in human inferior frontal cortex. Proc Nat Acad Sci 2010;107:13966-13971.

[40] West BT, Welch KB, Galecki AT. Linear Mixed Models: A practical guide using statistical software. London: Chapman \& Hall/CRC, 2007.

[41] Williams ACdeC. Facial expression of pain: an evolutionary account. Behav Brain Sci 2002;25:439-488.

[42] Wills TA. Perceptions of clients by professional helpers. Psychol Bull 1978;85:9681000. 


\section{Figure Captions}

Fig.1. Presentation of one trial during the acquisition phase; $\mathrm{CS}=$ conditioned stimulus, UCS $=$ unconditioned stimulus, ITI $=$ inter trial interval.

Fig.2. Observers' pain ratings as a function of valence of traits associated with patients and patients' pain expression. In the high intensity pain expression condition, a significant difference was found in observers' pain ratings when evaluating patients associated with negative traits versus neutral or positive traits. In the mild and no pain expression conditions, no differences were found. The intervals around condition means represent $95 \%$ confidence intervals. 


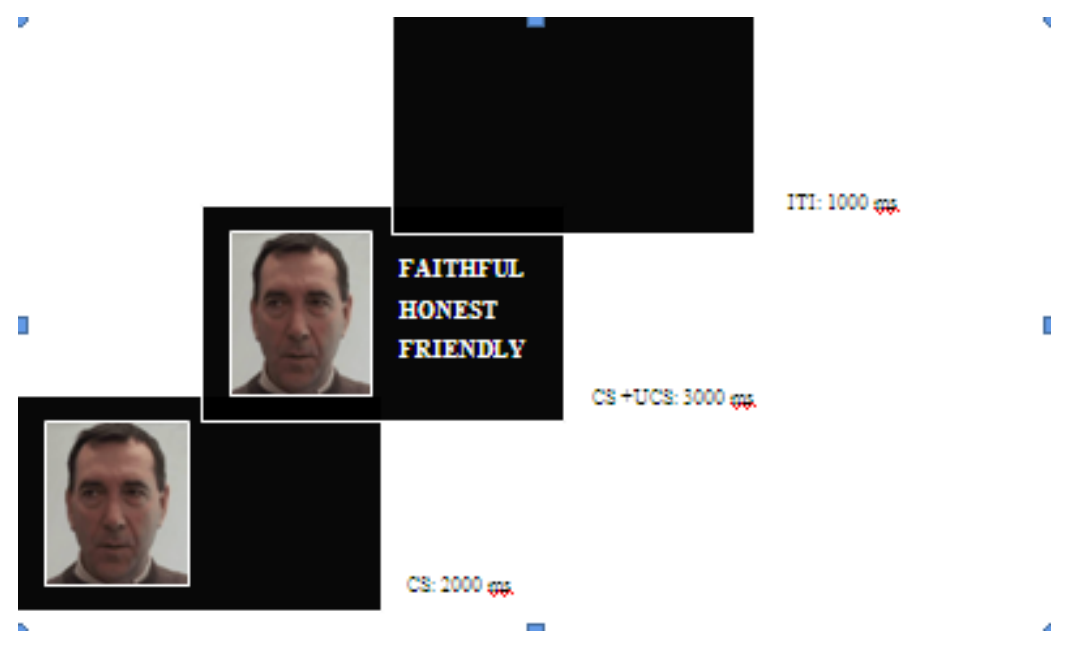




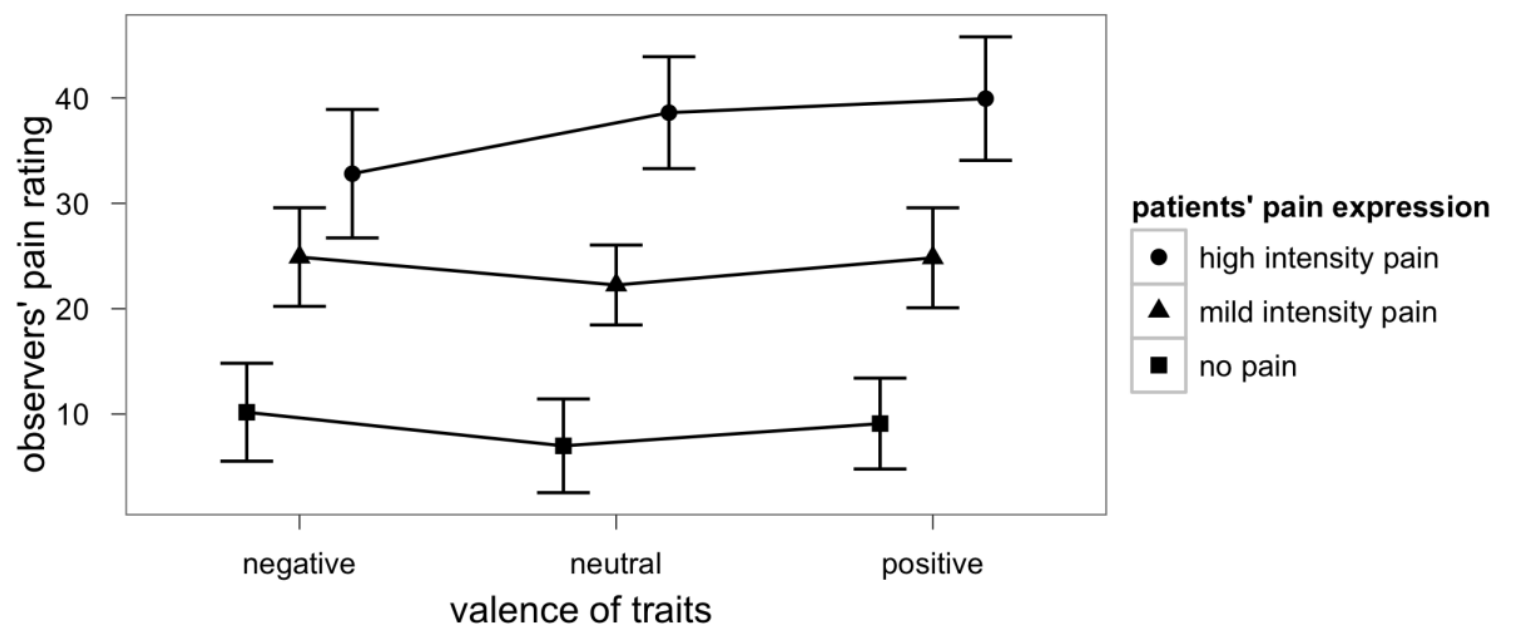

